\title{
The Digitation Sign Facilitates Diagnosis of Shoulder Subscapularis Lesions on Preoperative Magnetic Resonance Imaging
}

\author{
Ana Nigues, M.D., Yves Salentiny, M.D., Marko Nabergoj, M.D., \\ Alexandre Lädermann, M.D., and Lionel Neyton, M.D.
}

\begin{abstract}
Purpose: The purposes of this study were to investigate a radiographic sign found on coronal magnetic resonance imaging (MRI) sequences in subscapularis (SSC) pathology, reporting interobserver reliability data and sensitivity and specificity; and to correlate the preoperative assessment of SSC pathology with intraoperative assessment of the SSc during shoulder arthroscopy. Methods: A consecutive series of patients undergoing arthroscopic rotator cuff repair from January 2020 to December 2020 were examined. The positive diagnosis of a subscapularis tendon tear was prospectively determined and confirmed by arthroscopy. The "digitation sign" was assessed by 3 independent shoulder-trained fellows when evaluating the SSC in the coronal plane on T2-weighted images. Results: Of the 132 patients included, 74 (56\%) had SSC tendon tears confirmed during arthroscopy. Interobserver agreement for digitation sign was substantial $(\mathrm{k}=$ 0.640). Sensitivity for digitation sign only was 74.3\%; for criteria of Adams et al. (Accuracy of preoperative magnetic resonance imaging in predicting a subscapularis tendon tear based on arthroscopy; Arthroscopy 2010;26:1427-1433) only, $83.8 \%$; and for the combination of the 2 methods, $89.2 \%$. Specificity was $84.5 \%$ for digitation sign only, $77.6 \%$ for Adams et al. criteria only, and $67.2 \%$ for the combination of the 2 methods. Sensitivity is statistically better when the digitation sign is added to the Adams et al. criteria for detection of SSC lesions. As expected, specificity is statistically lower. SSC tears are significantly associated with biceps lesions. Conclusion: Preoperative systematic MRI evaluation by shoulder surgeons can diagnose subscapularis lesions by using the digitation sign in the coronal plane along with Adams et al. criteria, leading to good sensitivity. This easy-to-apply sign can be helpful in the initial MRI evaluation in the coronal plane, with improved sensitivity when combined with a systematic approach. Level of Evidence: II, study of diagnostic test.
\end{abstract}

From Pavillon E, Hôpital Edouard Herriot, Hospices Civils de Lyon, Lyon, France (A.N.); Groupe Orthopédique HRS, Hôpitaux Robert Schuman, Kirchberg, Luxembourg (Y.S.); Valdoltra Orthopaedic Hospital, Ankaran, Slovenia (M.N.); Faculty of Medicine, University of Ljubljana, Ljubljana, Slovenia (M.N.); Division of Orthopaedics and Trauma Surgery, La Tour Hospital, Meyrin, Switzerland (A.L.); Faculty of Medicine, University of Geneva, Geneva, Switzerland (A.L.); Division of Orthopaedics and Trauma Surgery, Department of Surgery, Geneva University Hospitals, Geneva, Switzerland (A.L.); Ramsay, Générale de Santé, Hôpital Privé Jean Mermoz, Lyon, France (L.N.); Centre Orthopédique Santy, Lyon, France (L.N.).

The authors report the following potential conflicts of interest or sources of funding: A.L. reports founder, BeeMed, Med4cast. L.N. reports personal fees, Wright, Arthrex. Full ICMJE author disclosure forms are available for this article online, as supplementary material.

Received September 12, 2021; accepted December 28, 2021.

Address correspondence to Lionel Neyton, Centre Orthopédique, Santy, 24 Avenue Paul Santy,69008Lyon, France.E-mail:neyton.lionel@orange.fr

(C) 2022 THE AUTHORS. Published by Elsevier Inc. on behalf of the Arthroscopy Association of North America. This is an open access article under the CC BY-NC-ND license (http://creativecommons.org/licenses/by-nc-nd/4.0/). 2666-061X/211288

https://doi.org/10.1016/j.asmr.2021.12.019
$\mathbf{R}$ eliable preoperative assessment of subscapularis (SSC) tears remains challenging because of limited sensitivity with physical examination maneuvers ${ }^{1,2}$ and preoperative imaging. ${ }^{3-5}$ Little inter- and intraobserver agreement is observed in the classification of subscapularis tendon tears, and there is significantly higher agreement based on arthroscopic evaluation rather than magnetic resonance imaging (MRI). ${ }^{6}$

Recognition of SSC tears on MRI is likely underestimated owing to the anatomy of the SSC insertion and its proximity to the ligamentous anatomy of the biceps pulley. ${ }^{7-9}$ The SSC has 4 to 6 tendinous slips arising from the multipennate muscle that coalesce to form a robust flattened tendon in the superior twothirds of the muscle, which attach to the lesser tuberosity. ${ }^{10-12}$ SSC tears are typically assessed on axial and sagittal sequences and can manifest with interrupted continuity of the tendon and signal hyperintensity on the T2-weighted images. ${ }^{11,13,14}$ Medial subluxation of the long head of the biceps tendon can also be 
suggestive of SSC pathology, ${ }^{8,13,15}$ and improvement is needed in MRI techniques. ${ }^{14}$

To improve the diagnostic accuracy and reliability of SSC tears based on first-time MRI analyses, various systematic approaches and sequences have been attempted. ${ }^{13,16,17}$ Adams et al. ${ }^{16}$ used axial and sagittal MRI images to define a set of criteria to more reliably diagnose SSC tears confirmed during shoulder arthroscopy. Although this approach improved their previously reported recognition of SSC pathology, 27\% of SSC tears were still not identified on MRI.

The purposes of this study were to investigate a radiographic sign found on coronal magnetic resonance imaging (MRI) sequences in subscapularis (SSC) pathology, reporting interobserver reliability data and sensitivity and specificity, and to correlate the preoperative assessment of SSC pathology with an intraoperative assessment of the SSC during shoulder arthroscopy. We hypothesized that, in the presence of SSC pathology, the normal ${ }^{4-6}$ distinct tendinous slips of SSC defining clear digitations would be disrupted on T2-weighted coronal oblique sequences.

\section{Methods}

\section{Study Design}

This is a retrospective analysis of a prospectively enrolled series of consecutive patients undergoing arthroscopic rotator cuff repair performed by a single surgeon (L.N.) from January 2020 to December 2020. The study was conducted according to the Declaration of Helsinki principles and was approved by the local institutional ethics review board (IRB COS-RGDS2020-12-005); all patients gave informed written consent. The inclusion criteria required that all patients had a preoperative MRI within 18 months of surgery, were age $>18$ years, and underwent primary arthroscopic rotator cuff repair. Furthermore, although several different MRI protocols were used by referring physicians, all protocols needed to have $\mathrm{T} 2$-weighted coronal oblique, axial, and sagittal oblique sequences and T1weighted sequences to be included. Correct sequences were extracted and validated by 1 fellow (A.N.) from preoperative DICOMS, and then interpreted by the 2 other fellows (Y.S., M.N.). The positive diagnosis of a subscapularis tendon tear was prospectively determined by the senior surgeon and confirmed by arthroscopy. Patient demographics included age, sex, and operative side.

\section{MRI Interpretation}

All MRI scans were interpreted independently by 3 orthopedic fellows trained in shoulder surgery, who were blinded from the arthroscopic findings. The fellows were trained by the senior surgeon and instructed in a systematic method ${ }^{16}$ upon their arrival at the

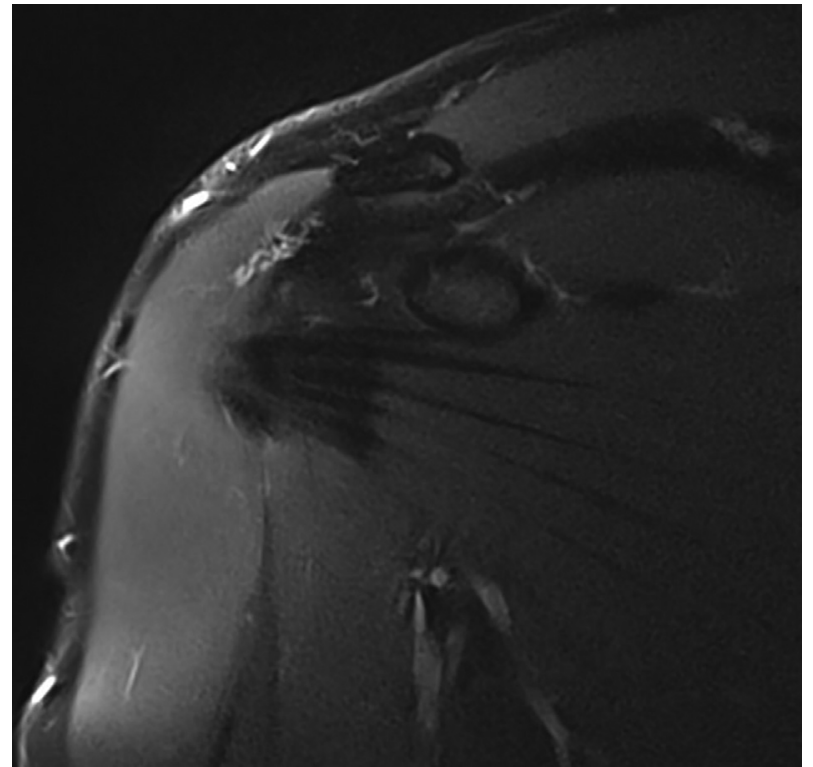

Fig 1. Coronal magnetic resonance imaging (MRI) subscapularis view in right shoulder. No lesion is suspected with complete digitations; digitation sign is considered negative.

fellowship. First, images were analyzed together until $100 \%$ agreement was reached between the senior surgeon and the fellow so that the latter could perform their analyses individually. DICOMS were examined with RadiAnt DICOM Viewer 2020.2.2.

According to Adams et al. ${ }^{16}$ criteria, the SSC was evaluated in axial, sagittal oblique, and coronal oblique planes. Tears were considered positive if the continuity of the subscapularis tendon was interrupted or signal hyperintensity was present on the $\mathrm{T} 2$-weighted images inside the tendon structure. In addition, tears of the involved rotator cuff tendons were recorded descriptively. To be close to real-life conditions, every MRI protocol meeting the above requirements was accepted.

The "digitation sign" was assessed when evaluating the SSC in the coronal plane on T2-weighted images. A normal appearance was defined as having $\geq 2$ distinctly visible tendinous bands ( 1 superior and 1 inferior) that coalesced into a single anatomic structure all the way to the proximal aspect of the lesser tuberosity (Fig. 1). Additional inferior tendinous bands could be observed. If $\geq 1$ (typically superior) tendinous bands were not visible at the level of the lesser tuberosity on T2weighted coronal oblique images, the digitation sign was defined as positive (Fig. 2). A normal appearance of the SSC with all its digitations was considered a negative digitation sign.

\section{Arthroscopic Procedure}

All patients underwent primary arthroscopic rotator cuff repair by a senior shoulder surgeon (L.N.). Shoulder arthroscopy was performed in the beach chair position under general anesthesia after preoperative 

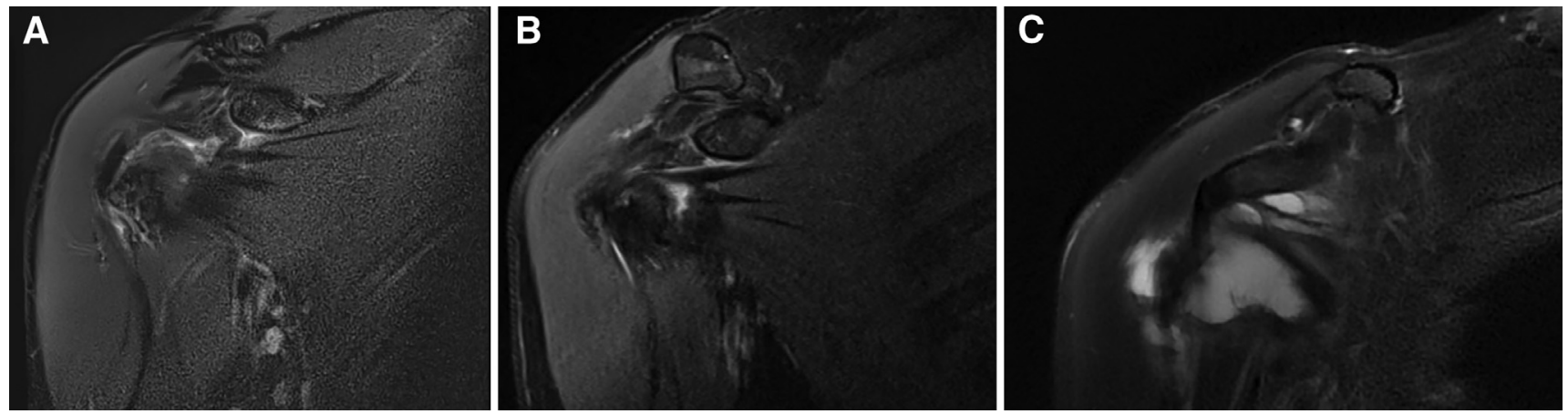

Fig 2. Coronal magnetic resonance imaging (MRI) subscapularis view in right shoulders. Subscapularis lesions are suspected with incomplete digitations; digitation sign is considered positive. A, grade II; B, grade III; C, grade IV. Grades were defined perioperatively according to French Society for Arthroscopy (SFA) and Lafosse et al. classifications.

interscalene block. Standard diagnostic arthroscopy was accomplished through a posterior viewing portal. Evaluation of the SSC was carried out with a $30^{\circ}$ arthroscope as previously described. ${ }^{18}$ SSC tears were classified according to the description of Lafosse et al. ${ }^{19}$ and the French Society for Arthroscopy (SFA) classification. $^{20}$ This measurement represented an ordinal measure of subscapularis tendon tear size and is based on the proximal-to-distal distance. In the case of a "hidden" lesion, the biceps gutter was debrided for later biceps tenodesis, as previously described. ${ }^{4}$ Concomitant shoulder pathology, including glenohumeral joint arthritis, biceps pathology, and labral lesions, was also reported. In the setting of biceps pathology, a suprapectoral tenodesis was performed with an interference screw. $^{21}$

\section{Sample Size Estimation}

We performed the sample size estimation using proportion comparison test to fix a proportion of $70 \%,{ }^{16}$ targeting a statistical power of $80 \%$ with a significance level of $5 \%$ and an estimated prevalence of $50 \%$. A sample of $\geq 122$ shoulders was determined. Considering sample loss, we decided to include 132 shoulders.

\section{Statistical Analysis}

Statistical analysis was conducted comparing SSC tendon tears recognized during shoulder arthroscopy (gold standard) to those diagnosed preoperatively by MRI. In addition, descriptive data analysis was conducted depending on the nature of the considered criteria. For quantitative data, this included number of observed values, mean, standard deviation, median, and minimum and maximum. For qualitative data, this included the number of observed values and the number and percentage of patients per class. Comparison between variables was assessed using chi-squared or Fisher's exact test for categorical variables and Student's $t$ test or Wilcoxon test for quantitative variables. Normality of variables was checked. Fleiss's $\kappa$ coefficient and standard error were calculated to measure interobserver agreement between 3 raters. The values of $\kappa$ were interpreted as follows: $<0$, poor agreement; 0.01 to 0.20 , slight agreement; 0.21 to 0.40 , fair agreement; 0.41 to 0.60 , moderate agreement; 0.61 to 0.80 , substantial agreement; and 0.81 to 1.00 , almost perfect agreement. Comparisons for sensitivity and specificity were performed with a McNemar test. All calculations were made with SAS for Windows (version 9.4; SAS Institute, Cary, NC), with the level of statistical significance set at $P<.05$.

\section{Results}

\section{Population Characteristics}

Of the 197 patients enrolled, 43 had a computed tomography (CT) arthrogram preoperatively, 21 had no complete available DICOM series, and 1 had previous surgery on the examined shoulder. Sixty-five patients were therefore excluded from the study. Of the 132 patients included, $74(56 \%)$ had SSC tendon tears confirmed during arthroscopy. The demographics of the patients are presented in Table 1. The groups were similar.

\section{Interobserver Reliability}

The interobserver analysis is presented in Table 2 . Interobserver agreement for digitation sign was substantial, with a $\kappa$ coefficient of 0.640 .

\section{Sensitivity and Specificity Analysis}

Fifty-five $(74.3 \%)$ SSC lesions were correctly diagnosed with digitation sign only, $62(83.8 \%)$ with Adams et al. ${ }^{16}$ criteria only, and $66(89.2 \%)$ with the combination of the 2 methods. Forty-nine $(84.5 \%)$ intact SSC tendons were correctly diagnosed with digitation sign only, $45(77.6 \%)$ with Adams et al. ${ }^{16}$ criteria only, and $39(67.2 \%)$ with the combination of the 2 methods. Sensitivity is statistically better when the digitation sign is added to the Adams et al. ${ }^{16}$ criteria for detection of SSC injury. As expected, specificity is 
Table 1. Demographic characteristics

\begin{tabular}{|c|c|c|c|c|}
\hline Characteristic & Entire Population & SSC Tears Group & Intact SSC group & $P$ Value \\
\hline $\mathrm{n}$ & 132 & 74 & 58 & \\
\hline Sex & & & & .214 \\
\hline Female & 48 & 23 & 25 & \\
\hline Age (years) & $58(28,82)$ & $59(41,82)$ & $57(28,78)$ & .181 \\
\hline Operated side & & & & .956 \\
\hline Right & 85 & 48 & 37 & \\
\hline Right & 127 & 71 & 57 & \\
\hline Left & 4 & 3 & 1 & \\
\hline $\begin{array}{l}\text { Delay between } \\
\text { presentation and } \\
\text { surgery }(\mathrm{mo})\end{array}$ & $3.1(0,18)$ & $2.7(0,11)$ & $3.5(0,18)$ & .181 \\
\hline
\end{tabular}

statistically lower. Sensitivity and specificity combinations are shown in Table 3.

\section{Accuracy According to Lesion Classifications}

The accuracy of the digitation sign and Adams et al. ${ }^{16}$ criteria in relation to the Lafosse et al. ${ }^{19}$ and SFA ${ }^{20}$ classifications are shown in Table 4 . Adams et al. ${ }^{16}$ criteria were more accurate than digitation sign in diagnosing lower-grade SSC lesions; however, both methods combined yielded the highest sensitivity.

\section{Concomitant Pathology}

Concomitant pathologies during arthroscopic exploration are shown in Table 5. SSC tears were significantly associated with biceps lesions.

\section{Discussion}

The principal findings of this study show that preoperative systematic MRI evaluation by orthopedic surgeons can successfully diagnose subscapularis lesions by using the digitation sign in the coronal plane in conjunction with Adams et al. ${ }^{16}$ criteria, leading to increased sensitivity.

Subscapularis tears are common shoulder lesions, but diagnostic accuracy based on physical examination remains poor. ${ }^{1,3}$ These tears are technically demanding for arthroscopic surgeons in both diagnosis and repair. ${ }^{4,22}$ An accurate and reliable imaging diagnosis is desirable to adapt surgical treatment strategy. ${ }^{9}$ The present study describes a simple MRI sign in the coronal plane, the digitation sign, with substantial interobserver reliability, good proper specificity, and improved sensitivity when added to Adams et al. ${ }^{16}$ criteria, confirming our hypothesis.

Recent advances in preoperative imaging and operative techniques have improved the ability to diagnose and treat subscapularis tendon tears, ${ }^{19,23}$ with good results. ${ }^{24}$ Coincidentally, the incidence of SSC tendon tears recognized and treated at the time of arthroscopy has increased substantially. ${ }^{22,23}$ It is important to note that intraoperative assessment of partial SSC tears can often be challenging to identify and have therefore been referred to as "hidden lesions." ${ }^{\text {"14,25 }}$ Numerous MRI signs have been investigated to diagnose subscapularis lesions, ${ }^{13,26-30}$ with often good or excellent specificity but lack of sensitivity. The first ones compared MRI diagnosis to open or arthroscopic procedures, ${ }^{28,29}$ but the superiority of arthroscopic techniques to determine SSC tears, particularly on the articular side, is well described in the literature. .,7,14,23 $^{2}$ Adams et al. ${ }^{13}$ found an excellent specificity of $100 \%$ in 52 SSC tears in 120 patients when diagnosed by radiologists. With a standardized approach to MRI reading, however, they found similar results between surgeons and radiologists regarding the accuracy in diagnosing SSC lesions, provided that the radiologists were musculoskeletal radiologists in majority. ${ }^{16}$ The best scenario for subscapularis tear detection consists of 2 musculoskeletal radiologists evaluating every preoperative MRI, ${ }^{28,31}$ but such complex imaging procotols $^{17,32}$ are rare in daily practice. The medicolegal aspect of imaging interpretation should also be considered in professional pathologies. Baptista et al. ${ }^{26}$ noted that an unsure radiologist or surgeon might not express

Table 2. Interobserver analysis

\begin{tabular}{|c|c|c|c|c|c|c|}
\hline Item & Adams et al. Criteria $^{16}$ & Axial Injury & Biceps Subluxation & Sagittal Injury & Atrophy & Digitation sign \\
\hline $\bar{\kappa}$ Coefficient & 0.513 & 0.507 & 0.251 & 0.579 & 0.203 & 0.640 \\
\hline Agreement & Moderate & Moderate & Fair & Moderate & Slight & Substantial \\
\hline
\end{tabular}


Table 3. Sensitivity analysis

\begin{tabular}{lcccc}
\hline Test attribute & Digitation Sign (\%) & Adams et al. Criteria ${ }^{16}(\%)$ & Digitation sign + Adams et al. Criteria $(\%)$ & $P$ Value \\
\hline Sensitivity & 74.3 & 83.8 & 89.2 & .046 \\
Specificity & 84.5 & 77.6 & 67.2 & .014 \\
\hline
\end{tabular}

their doubts and be elusive in the report, which can lead to the exclusion of most challenging cases, resulting in bias and overestimating diagnostic measures.

Despite the high specificity reported, Adams et al. ${ }^{13}$ found a sensitivity of $36 \%$ in the same series of 120 patients. This low sensitivity is consistent with the current literature. ${ }^{9,29}$ The importance of using multiple imaging planes underlines the necessity of systematics approaches to improve sensitivity. ${ }^{31,33}$ With a systematic approach to imaging and a combination of 7 criteria, Lin et al. ${ }^{34}$ observed an overall sensitivity of $82.2 \%$; specificity of $92.1 \%$; positive and negative predictive values of $87.1 \%$ and $88.9 \%$, respectively; and accuracy of $88.2 \%$. They did not perform an interobserver analysis allowing for comparison and reproducibility of the data collected from analyzing the preoperative imaging.

The digitation sign by itself has a sensitivity of $74.3 \%$ and a specificity of $84.5 \%$, based on shoulder-trained fellows' analysis. However, our systematic approach, combining digitation sign and Adams et al. ${ }^{16}$ criteria, showed a significantly improved sensitivity of $89.2 \%$. In our study, the sensitivity of Adams et al. ${ }^{16}$ criteria was higher $(83.8 \%)$ than in the original cohort $(73 \%),{ }^{13}$ possibly because of prevalence variations.

Prevalence of SSC lesions, which influences sensitivity analysis, was higher in our population than previously reported, ${ }^{3,11,13,16,31,35}$ with $56 \%$ having SSC tears. This can be explained by the attention of the senior surgeon to hidden lesions and systematically looking for SSC tears associated with biceps lesions by opening the biceps gutter. $^{23}$ Grade III or IV SSC tears according SFA ${ }^{20}$ or Lafosse et al. ${ }^{19}$ classification are easily diagnosed, with $94.7 \%$ of cases presenting a positive digitation sign, $100 \%$ positive for Adams et al. ${ }^{16}$ criteria, and $100 \%$ for a combination of both signs, which is consistent with previous studies. ${ }^{14,16,17,31}$ Lin et al. ${ }^{34}$ also concluded that partial-thickness tears (less than one third of tendon width) were easily missed, and that there was increased sensitivity to the type of lesion, with a $100 \%$ sensitivity for type IV and V lesions according to Lafosse et al. ${ }^{19}$

We suggest using the digitation sign as a quick firstlook tool during MRI analysis because of the difficulty in diagnosing small SSC lesions (Lafosse et al. I or II and SFA 1 or 2)..$^{8,13,14,17}$ All other signs should be checked in a systematic procedure for meticulous analysis. The results of Lin et al. ${ }^{34}$ are in accordance with the idea that SSC tears can be diagnosed with several systematic criteria, obtaining the best sensitivity.

Lack of homogeneity may be closer to the daily practice of most shoulder surgeons with patients being referred with different MRIs. Our results are consistent with other similar studies in the literature, ${ }^{6,26,33}$ however, showing that the analysis of MRIs depends strongly on the observer. In contrast, our systematic approach to MRI interpretation is reproducible $(\mathrm{k}=$ 0.640 ) and can be used with standard MRI protocols of different institutions in daily practice. This protocol was designed with shoulder-trained fellows and can be helpful for young surgeons in their preoperative evaluation. Our higher SSC tear prevalence $(56 \%)$ may also reflect a systematic arthroscopic evaluation for hidden lesions.

\section{Limitations}

This study had several limitations. First, the analysis was retrospective. There was an 18-month delay between MRI and surgery. Furthermore, there were only 3 shoulder trained fellows from different institutions but no radiologist interpreting the MRIs, and there was no intraobserver agreement analysis. Different MRI protocols were used in each radiology institution,

Table 4. Detection of subscapularis (SSC) tears according to lesion grade

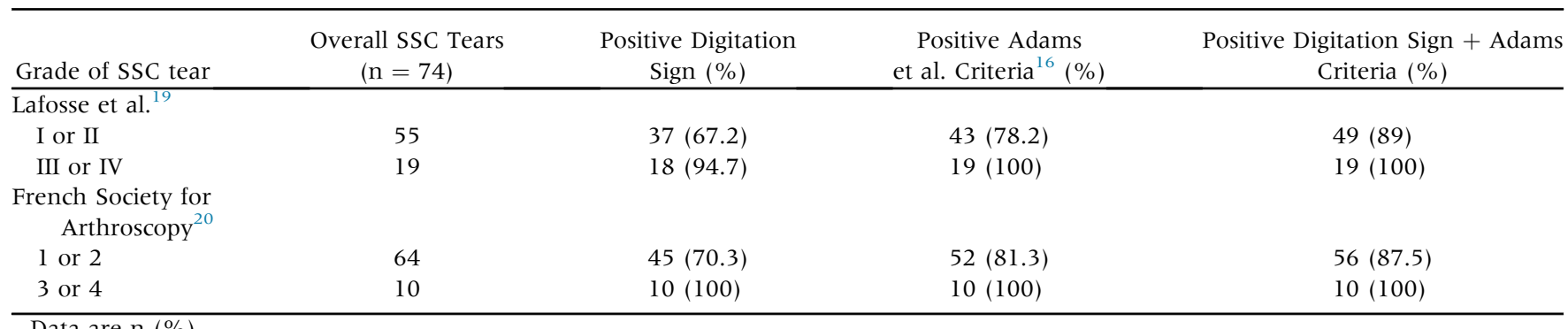

Data are n $(\%)$. 
Table 5. Concomitant lesions

\begin{tabular}{lccc}
\hline \multicolumn{1}{c}{ Lesions } & Entire Population $(\mathrm{n}=132)$ & SSC Tears Group $(\mathrm{n}=74)$ & Intact SSC Group $(\mathrm{n}=58)$ \\
\hline Supraspinatus & 128 & 71 & 57 \\
Infraspinatus & 109 & 61 & .630 \\
Biceps & 83 & 57 & .855 \\
Old biceps rupture & 9 & 6 & 26 \\
\hline
\end{tabular}

Abbreviation: SSC, subscapularis.

which might impair MRI analysis. Our interobserver analysis reveals a globally poor interevaluation between the 3 observers.

\section{Conclusions}

Preoperative systematic MRI evaluation by shoulder surgeons can diagnose subscapularis lesions by using the digitation sign in the coronal plane with Adams et al. ${ }^{16}$ criteria, leading to good sensitivity. This easy-toapply sign can be helpful in the initial MRI evaluation in the coronal plane, with improved sensitivity when combined with a systematic approach.

\section{Acknowledgments}

FORE (Foundation for Research and Teaching in Orthopaedics, Sports Medicine, Trauma and Imaging in the Musculoskeletal System), grant number FORE 2021-13.

\section{References}

1. Gerber C, Krushell RJ. Isolated rupture of the tendon of the subscapularis muscle. Clinical features in 16 cases. J Bone Joint Surg Br 1991;73:389-394.

2. Hegedus EJ, Goode A, Campbell S, et al. Physical examination tests of the shoulder: A systematic review with meta-analysis of individual tests. Br J Sports Med 2008;42: 80-92.

3. Barth JRH, Burkhart SS, De Beer JF. The bear-hug test: A new and sensitive test for diagnosing a subscapularis tear. Arthroscopy 2006;22:1076-1084.

4. Burkhart SS, Brady PC. Arthroscopic subscapularis repair: Surgical tips and pearls A to Z. Arthroscopy 2006;22: 1014-1027.

5. Teefey SA, Rubin DA, Middleton WD, Hildebolt CF, Leibold RA, Yamaguchi K. Detection and quantification of rotator cuff tears. Comparison of ultrasonographic, magnetic resonance imaging, and arthroscopic findings in seventy-one consecutive cases. J Bone Joint Surg Am 2004;86:708-716.

6. Smucny M, Shin EC, Zhang AL, et al. Poor agreement on classification and treatment of subscapularis tendon tears. Arthroscopy 2016:32:246-251.el.

7. Eren I, Ozben H, Gunerbuyuk C, et al. Rotator cuff tear characteristics: How comparable are the pre-operative MRI findings with intra-operative measurements following debridement during arthroscopic repair? Int Orthop 2019;43:1171-1177.
8. Shi LL, Mullen MG, Freehill MT, Lin A, Warner JJP, Higgins LD. Accuracy of long head of the biceps subluxation as a predictor for subscapularis tears. Arthroscopy 2015;31:615-619.

9. Vinson EN, Wittstein J, Garrigues GE, Taylor DC. MRI of selected abnormalities at the anterior superior aspect of the shoulder: Potential pitfalls and subtle diagnoses. AJR Am J Roentgenol 2012;199:534-545.

10. Arai R, Mochizuki T, Yamaguchi K, et al. Functional anatomy of the superior glenohumeral and coracohumeral ligaments and the subscapularis tendon in view of stabilization of the long head of the biceps tendon. J Shoulder Elbow Surg 2010;19:58-64.

11. Morag Y, Jamadar DA, Miller B, Dong Q, Jacobson JA. The subscapularis: Anatomy, injury, and imaging. Skeletal Radiol 2011;40:255-269.

12. Yoo JC, Rhee YG, Shin SJ, et al. Subscapularis tendon tear classification based on 3-dimensional anatomic footprint: A cadaveric and prospective clinical observational study. Arthroscopy 2015;31:19-28.

13. Adams CR, Schoolfield JD, Burkhart SS. Accuracy of preoperative magnetic resonance imaging in predicting a subscapularis tendon tear based on arthroscopy. Arthroscopy 2010;26:1427-1433.

14. Foad A, Wijdicks CA. The accuracy of magnetic resonance imaging and magnetic resonance arthrogram versus arthroscopy in the diagnosis of subscapularis tendon injury. Arthroscopy 2012;28:636-641.

15. French Society for Arthroscopy (SFA), Godenèche A, Nové-Josserand L, et al. Relationship between subscapularis tears and injuries to the biceps pulley. Knee Surg Sports Traumatol Arthrosc 2017;25:2114-2120.

16. Adams CR, Brady PC, Koo SS, et al. A systematic approach for diagnosing subscapularis tendon tears with preoperative magnetic resonance imaging scans. Arthroscopy 2012;28:1592-1600.

17. Furukawa R, Morihara T, Arai Y, et al. Diagnostic accuracy of magnetic resonance imaging for subscapularis tendon tears using radial-slice magnetic resonance images. J Shoulder Elbow Surg 2014;23:e283-e290.

18. Gasbarro G, Neyton L. The arthroscopic "montgolfier double-row knotless" rotator cuff repair technique. Arthrosc Tech 2019;8:e669-e674.

19. Lafosse L, Lanz U, Saintmard B, Campens C. Arthroscopic repair of subscapularis tear: Surgical technique and results. Orthop Traumatol Surg Res 2010;96:S99-S108.

20. Toussaint B, Barth J, Charousset C, et al. New endoscopic classification for subscapularis lesions. Orthop Traumatol Surg Res 2012;98:S186-S192.

21. Amouyel T, Le Moulec YP, Tarissi N, Saffarini M, Courage O. Arthroscopic biceps tenodesis using 
interference screw fixation in the bicipital groove. Arthrosc Tech 2017;6:e1953-e1957.

22. Lo IKY, Burkhart SS. The comma sign: An arthroscopic guide to the torn subscapularis tendon. Arthroscopy 2003;19:334-337.

23. Neyton L, Daggett M, Kruse K, Walch G. The hidden lesion of the subscapularis: Arthroscopically revisited. Arthrosc Tech 2016;5:e877-e881.

24. Denard PJ, Jiwani AZ, Lädermann A, Burkhart SS. Longterm outcome of a consecutive series of subscapularis tendon tears repaired arthroscopically. Arthroscopy 2012:28:1587-1591.

25. Walch G, Nove-Josserand L, Levigne C, Renaud E. Tears of the supraspinatus tendon associated with "hidden" lesions of the rotator interval. J Shoulder Elbow Surg 1994;3: 353-360.

26. Baptista E, Malavolta EA, Gracitelli MEC, et al. Diagnostic accuracy of MRI for detection of tears and instability of proximal long head of biceps tendon: An evaluation of 100 shoulders compared with arthroscopy. Skeletal Radiol 2019;48:1723-1733.

27. Li XX, Schweitzer ME, Bifano JA, Lerman J, Manton GL, El-Noueam KI. MR evaluation of subscapularis tears. J Comput Assist Tomogr 1999;23:713-717.

28. Pfirmann CW, Zanetti M, Weishaupt D, Gerber C, Hodler J. Subscapularis tendon tears: Detection and grading at MR arthrography. Radiology 1999;213:709-714.
29. Tung GA, Yoo DC, Levine SM, Brody JM, Green A. Subscapularis tendon tear: Primary and associated signs on MRI. J Comput Assist Tomogr 2001;25:417-424.

30. Zhang H, Zhang Q, Li ZL. Coracohumeral index and coracoglenoid inclination as predictors for different types of degenerative subscapularis tendon tears. Int Orthop (SICOT) 2019;43:1909-1916.

31. Gyftopoulos S, O’Donnell J, Shah NP, Goss J, Babb J, Recht MP. Correlation of MRI with arthroscopy for the evaluation of the subscapularis tendon: A musculoskeletal division's experience. Skeletal Radiol 2013;42:1269-1275.

32. Meyer DC, Zimmermann SM, Wieser K, Bensler S, Gerber C, Germann M. Lengthening of the subscapularis tendon as a sign of partial tearing in continuity. J Shoulder Elbow Surg 2016;25:31-37.

33. Ryu HY, Song SY, Yoo JC, Yun JY, Yoon YC. Accuracy of sagittal oblique view in preoperative indirect magnetic resonance arthrography for diagnosis of tears involving the upper third of the subscapularis tendon. J Shoulder Elbow Surg 2016;25:1944-1953.

34. Lin L, Yan H, Xiao J, et al. The diagnostic value of magnetic resonance imaging for different types of subscapularis lesions. Knee Surg Sports Traumatol Arthrosc 2016;24:2252-2258.

35. Bennett WF. Subscapularis, medial, and lateral head coracohumeral ligament insertion anatomy. Arthroscopy 2001;17:173-180. 